\title{
A Simple Approach for Coupling Liquid Chromatography and Electron Ionization Mass Spectrometry
}

\author{
Achille Cappiello, Giorgio Famiglini, Filippo Mangani, and \\ Pierangela Palma \\ Istituto de Scienze Chimiche "F. Bruner," Università di Urbino, Urbino, Italy
}

\begin{abstract}
A miniaturized, aerosol based interface for directly coupling a liquid chromatograph with a mass spectrometer is presented. The interface is entirely within the electron ionization (EI) source of the mass spectrometer and no additional, external devices are needed. This simple and effective approach exploits micro-flow nebulization technology providing a new interface suitable for a variety of applications of environmental and biological interest. The new interface provides necessary linearity, ruggedness, sensitivity, and reproducibility of response for trace level analysis, and readily interpretable mass spectra for unambiguous identification of unknown compounds of small to medium molecular weight. (J Am Soc Mass Spectrom 2002, 13, 265-273) (c) 2002 American Society for Mass Spectrometry
\end{abstract}

$\mathrm{H}$ igh performance liquid chromatography (HPLC) and electron ionization mass spectrometry (EIMS) play a major role in their respective fields, solving a variety of analytical problems. They are the extremes of two different technical approaches: The first one typically employs very high pressure for the efficient separation of analytes dissolved in a sometimes complex liquid phase; the second one, a sophisticated detector for chromatography, measures the mass-to-charge ratio of the analytes and operates at a very high vacuum, a rarefied gas phase with no tolerance for extraneous substances. The characteristics of HPLC and EIMS are often seen as incompatible: The effort of achieving and maintaining the high vacuum required for mass spectrometry operation is in contrast with the intrinsic nature of HPLC, predominantly operating at high solvent flow rates. In addition, the low tolerance of mass spectrometers for non-volatile mobile phase components contrasts with a HPLC dependence on non-volatile buffers to achieve high-resolution separations. The two techniques, which in principle appear totally incompatible, may nevertheless show an impressive number of overlapping applications. Small to medium sized molecules, typically under $1000 \mathrm{u}$, that for several reasons are suitable for HPLC analysis, can generate highly informative, reproducible, library matchable EI mass spectra for an easier identification and characterization. Thousands of these compounds can be found in many different areas of

Published online January 14, 2002

Address reprint requests to Prof. A. Cappiello, Istituto di Scienze Chimiche, Università di Urbino, Piazza Rinascimento 6, 61029 Urbino, Italy. E-mail: achille@uniurb.it environmental, biological, or pharmaceutical interest. Therefore, a single analytical tool that combines the two techniques without limiting their potential is highly desirable. Today, most of the commercially available HPLC/MS interfaces are based on the atmospheric pressure ionization technique (API), namely electrospray (ESI) and atmospheric pressure chemical ionization (APCI) $[1,2,3,4]$ in which the ionization of the sample is obtained at atmospheric pressure in the liquid phase and only the ionic species are then admitted into the high vacuum analyzer region. This particular ionization process is considered soft in terms of internal energy, with no fragmentation normally observed. Polarity is a key factor for a satisfying response of ESI and APCI interfaces. Electron ionization, because of the higher internal energy involved, produces several neutral and ionic fragments in a peculiar combination of abundance and mass-to-charge ratio characteristic of each compound as a real chemical fingerprint. EI itself is not influenced by the polarity of the analytes. EI detection can also benefit from a recent sophisticated algorithm developed by the National Institute of Standards and Technology (NIST) called AMDIS (Automated MS Deconvolution and Identification System), which extracts the analytes mass spectra in complex chromatographic mixtures even with overlapping peaks, and further exploits the full potential of EI. The unsurpassed identification capability of EI is valuable for the detection of unknowns or for the confirmation of a specific sample composition, but because of the high vacuum requirement, EI can be easily compromised by any solvent vapor residue of the mobile phase. An exhaustive discussion on this topic is reported in two recent review articles $[5,6]$. 
The first successful on-line combination of liquid chromatography and EI mass spectrometry dates back about fifteen years and was based on the conversion of the liquid effluent into an aerosol and, after the evaporation of the aerosol droplets and the elimination of the solvent vapors through a multi-stage momentum separator, transformed into a beam of solute particles. This first attempt was later called particle beam interface [7]. The performance of particle beam has a few, rather crucial, drawbacks: Poor sensitivity for water-rich mobile phases, poor linearity and sensitivity for polar compounds, low tolerance for heat sensitive compounds, and an unpredictable optimization procedure. Our research group introduced in 1993 [8] an up-to-date version of the interface in which the operating mobile phase flow rate was reduced to the nL-per-min range. To achieve this limit, the nebulizer was entirely redesigned replacing the one supplied by the manufacturer. The rest of the interface was left unmodified. The effect of the minimized solvent intake improved the level of performance of the particle beam interface. The latest version of this interface is called Capillary-EI (Cap-EI) and was recently developed by our group [9]. Cap-EI takes advantage of a complete re-engineering of each stage of the interface, to further improve the performance of this type of interface.

Kientz et al. [10] proposed a different approach for generating electron ionization spectra from a HPLC effluent based on an eluent-jet formation by means of inductive heating of the micro-LC effluent, and momentum separation in a jet separator. Dijkstra et al. [11] recently improved this technique with a particular emphasis on its use under chemical ionization CI-MS conditions. Amirav, whose contribution in the field of supersonic molecular beam mass spectrometry (SMBMS) is well recognized, is now developing a new apparatus for obtaining high quality, library-searchable EI spectra based on the SMB approach.

The aim of the work presented in this paper was to develop a much simpler, rugged, all-purpose interface, called Direct-EI interface, for the generation of high quality electron ionization spectra from a liquid effluent. Instead of developing another complex device to eliminate the mobile phase, we exploited the best of the micro-flow nebulization technology for a direct introduction of the eluate into the EI ion source [12]. The new approach benefits from the enormous efforts in the early days of LC/MS interfacing when the direct introduction of liquid effluent (DLI) first appeared [13-20]. However, DLI was affected by various problems such as frequent clogging of the transport capillary, unstable source pressure, and sample sputtering, which contributed to its fleeting success. In addition, the ionization was chemical in nature rather than produced by the mere impact of electrons because the flow rates available at that time rarely were below $10 \mu \mathrm{L} / \mathrm{min}$, and the relative pressure of the mobile phase vapors in the ion source was high enough to effect CI. It is worth pointing out that in contrast to DLI, Direct-EI takes advantage the reliability of micro- and nano-HPLC columns, so that the operating flow rate is low enough to allow prevention of any chemical ionization process. The ion source and the nebulizer used in the Direct-EI were designed for a rapid and complete removal of the solvent vapors, thus avoiding the typical DLI drawbacks. The new interface accomplished the following objectives:

1. Maximum simplicity. No complex mechanisms are involved.

2. High sensitivity. No sample loss due to the transport process.

3. Broad spectrum of application for low to medium weight molecules.

The performance of the Direct-EI interface was thoroughly evaluated with different test compounds and over a long period of time. Sensitivity, intra-day reproducibility, response linearity were determined and are better than anything previously reported for electron ionization. Detection limits were in low picogram range in selected ion monitoring (SIM) mode and in high picogram to low nanogram range in full scan mode for several analytes. The improved sensitivity of the Direct-EI interface can be especially appreciated when operating in full scan mode, since its major appeal relies on the outstanding identification capabilities of the full electron ionization spectrum.

\section{Experimental}

\section{HPLC/MS Interface}

The major obstacle encountered when a liquid effluent is introduced into the high vacuum of a mass spectrometer is represented by the immediate vaporization of the liquid and the subsequent instability of the high vacuum in the ion source housing. Generally, in any HPLC/MS interface, most of the effort is devoted to eliminating the solvent vapors from the different instrument compartments without interfering with the sample ionization, and eventually generating a solvent-free ion beam focused into the high vacuum analyzer region. Even in API based interfaces, where the liquid plays a fundamental role in the ionization process, the solvent has to be removed prior to the analyzer region. Electron ionization requires a solvent free environment necessitating the removal of the solvent early in the analysis procedure.

The recent development of a new class of capillaryscale HPLC columns has brought the liquid phase separations to a new dimension where complex mixtures can be separated at a microliter per min flow rate or less. A good micro-chromatography interface minimizes the impact of the solvents in the mass spectrometer without a loss in separation performance. Our research group has acquired much experience with aerosol formation at micro-scale flow rates and we used 


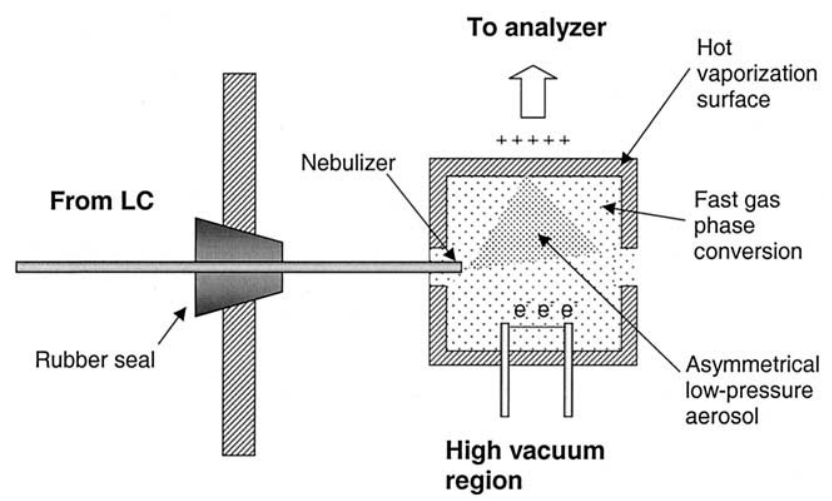

Figure 1. Schematic of the Direct-EI interface.

it in developing this interface. The originality consists in compressing all the interfacing process into the small volume of the electron ionization ion source of the mass spectrometer. The interface mechanism is based on the formation of the aerosol in high vacuum conditions, followed by a quick droplet desolvation and final vaporization of the solute prior to the ionization. The completion of the process requires less than $8 \mathrm{~mm}$ in space and only slight modifications of the original EI ion source as shown in Figure 1, which is a schematic of the interface. Fused silica capillary tubing connects the nebulizer with the capillary column, passing from an atmospheric pressure region to high vacuum. The total volume of the capillary does not affect the chromatographic separation and does not induce band broadening. The capillary tubing is thermally insulated to avoid in-tube vaporization of the mobile phase from the source heat. The aerosol jet is directed to a specific spot inside the ion source that acts as a hot vaporization surface. The hot, smooth stainless steel surface is at the same temperature as the rest of the ion source and converts the solute into the gas phase for subsequent ionization.

The principal component of the interface is the micro-nebulizer (Figure 2), which consists of a coneshaped tip slightly bent sideways (approximately $10^{\circ}$ ) and with an orifice of approximately $5 \mu \mathrm{m}$ (Figure 3). The nebulizer tip protrudes $2 \mathrm{~mm}$ into the ion source, and creates a backpressure that limits any premature, in-tube solvent evaporation. The emerging liquid phase is rapidly converted into concentrated aerosol droplets by the shape of the nebulizer tip, and pointed off-axis towards a specific target surface. The droplets are easily desolvated during the $8 \mathrm{~mm}$ long journey between the nebulizer and the source surface, producing even smaller solute particles directed to the target surface. The partial pressure of the solvent vapors is kept low by an additional opening in the ion volume. A mobile phase flow rate higher than $1.5 \mu \mathrm{L} / \mathrm{min}$ is larger than the conductance of this design and may lead to chemical ionization. The microphotograph shown in Figure 4 was taken after collecting the aerosol fingerprint on the surface for $5 \mathrm{~min}$ at a flow rate of $300 \mathrm{~nL} / \mathrm{min}$. The aerosol generation cannot be observed outside the sys-

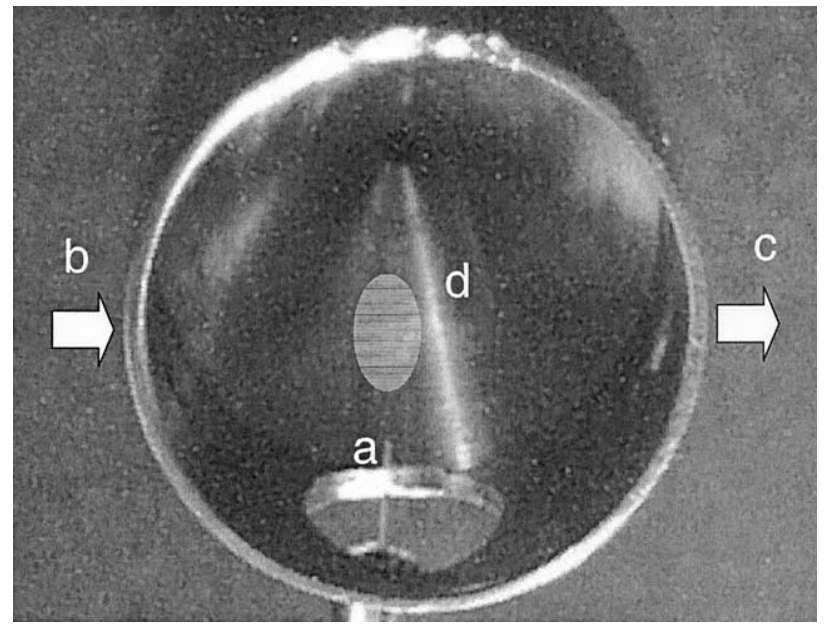

Figure 2. This figure shows a microphotograph of the interface taken from the analyzer side. The nebulizer tip (a) emerges $2 \mathrm{~mm}$ inside the ion source, which in this case, is of circular shape. The aerosol jet (a) is directed approximately towards the ellipsoidal spot (d) where the solute is immediately vaporized. The metal tubing, partially visible on the bottom of the picture, has the function of protecting the incoming liquid from the source heat. Letters $\mathbf{b}$ and $\mathbf{c}$ represent the points were the high energy electrons are introduced and removed respectively from the ion source. The gas phase molecules of the sample are produced right in sight of the electron beam for an easier and more efficient ionization. The cone shaped surface, where the vaporization is achieved, is used in the Hewlett-Packard mass spectrometer as an ion repeller.

tem because it is promoted by the combined effect of the high vacuum and the high temperature in the ion source.

The experiment proves that the liquid phase is converted into an aerosol prior to solvent evaporation. This is an important aspect of the interfacing mechanism: When the liquid effluent is converted into aerosol droplets, the total surface area of the eluate exposed to heat is much higher, speeding up the desolvation process. In addition, as a result of this process, the solute reaches the hot surface as a multitude of small molecular aggregates for an instant, decomposition-free va-

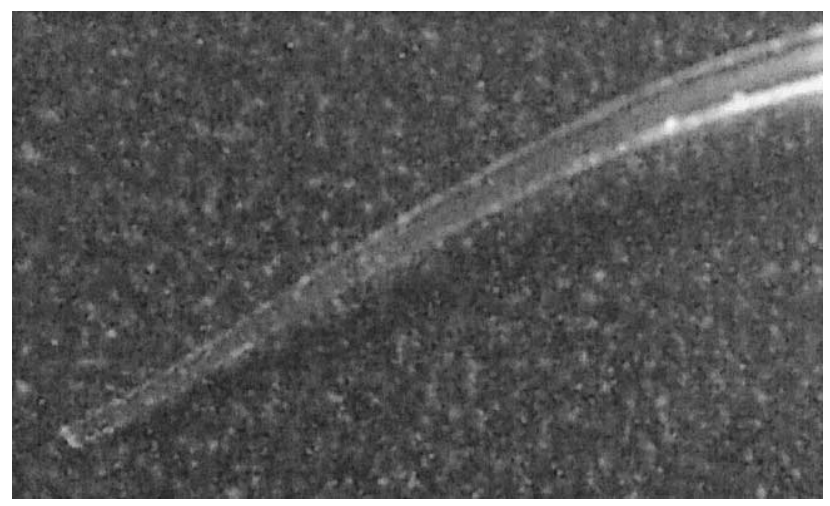

Figure 3. Microphotograph of the nebulizer tip. The bent shape, clearly visible in the picture, points the aerosol jet at the vaporization surface. The restricted orifice of the tip increases the liquid linear velocity and eases the aerosol formation under vacuum conditions. 


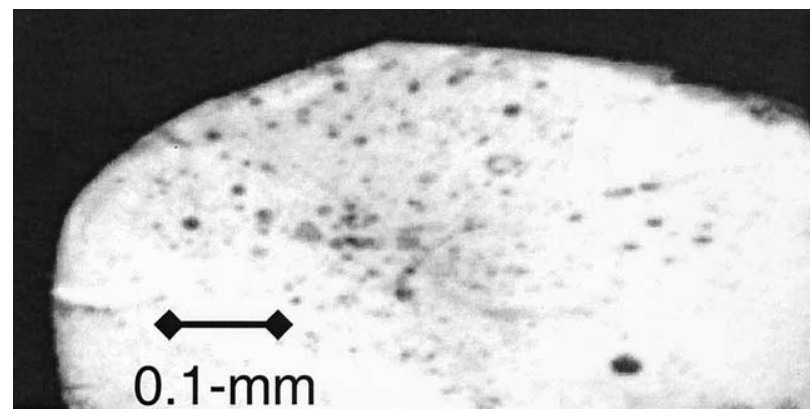

Figure 4. This figure shows a microphotograph of the aerosol fingerprints collected on the target surface of the ion source. A 10 $\mathrm{ng} / \mu \mathrm{L}$ solution of caffeine was continuously injected into the system for $5 \mathrm{~min}$ at a flow rate of $300-\mathrm{nL} / \mathrm{min}$.

porization. The spots visible on the photograph circumscribe a pretty well defined area a few millimeters wide in which the aerosol origin is clearly identifiable. The presence of larger or darker spots may indicate a wide distribution of the particle size or the superimposition of multiple particles on the same point.

While the aerosol formation is the key to the interface process, the high temperature of the ion source, between 150 and $300{ }^{\circ} \mathrm{C}$, has a double function: To compensate for the latent heat of vaporization during the droplet desolvation, and to convert the solute into the gas phase upon contact on the hot target surface. Temperature also prevents the liquid from freezing in the nebulizer, although the nano-flow rate used facilitates the vaporization and helps avoid mobile phase condensation.

The nebulizer is connected to the analytical microcolumn via a 30 or $50 \mu \mathrm{m}$ i.d. capillary tubing, passing from an atmospheric pressure region to a high vacuum one. To avoid in-tube vaporization of mobile phase as a result of the radiating source heat, the capillary tubing is thermally insulated. The whole liquid passage is kept well insulated from the source heat to avoid any premature mobile phase vaporization and possible solute degradation. All these features, together with the nebulizer backpressure, contribute to keeping the mobile phase as a liquid up to the nebulizer tip. The aerosol jet is precisely directed off-axis to a specific target surface inside the ion source. The hot, smooth stainless steel surface converts the solute into the gas phase for subsequent ionization. An additional opening in the ionization source, opposite to the nebulizer tip, increases solvent vapor removal, helping avoid ion-molecule reactions. Neither the electron path nor the electric fields are influenced by the interface intrusion into the ion volume and high quality mass spectra are thus produced. The tip of the nebulizer can be replaced to accommodate different mobile phase flow rates. The lowest amount required for generating a fine and homogeneous aerosol is $100 \mathrm{~nL} / \mathrm{min}$. The highest flow rate compatible with the interface is approximately 1.5 $\mu \mathrm{L} / \mathrm{min}$ and depends on the solvent vapor removal capability of the ion source design.
The major advantage of this technical solution is the extreme compactness and simplicity without any need for additional complex devices between the liquid chromatograph and the mass spectrometer. The lack of any particular transport mechanism involved in the interfacing process reduces sample losses enhancing sensitivity and extending the range of possible applications. In other words, the interfacing process has a lower influence on the sample transition and the only limitation is represented by the applicability of the electron ionization.

\section{Mass Spectrometry}

The new interface was coupled with a Hewlett-Packard 5989A quadrupole mass spectrometer. The operating pressure was approximately 8 to $10 \times 10^{-5}$ torr measured in the manifold of the ion source at the highest operating flow rate $(1.5 \mu \mathrm{L} / \mathrm{min})$, using a mixture composed of equal amounts of water and acetonitrile. Mass spectrometer tuning and calibration were performed automatically using perfluorotributylamine (PFTBA) as a reference compound and monitoring $\mathrm{m} / \mathrm{z}$ $69,219,502$. Considering the average molecular weight of our test compounds, the repeller potential was adjusted manually and optimized for the $\mathrm{m} / \mathrm{z} 219$ signal. No mobile phase was allowed into the ion source during calibration. The dwell times during SIM and scan times for full spectrum acquisition were adjusted to obtain 0.5 cycles/s and a mean of 10 acquisition samples for each HPLC peak. One- to three-ion detection was used in SIM mode. The final transfer tube prior to the ion source, was shifted to a fully retracted position after the tuning procedure allowing an additional pumping stage for a more efficient solvent vapor removal.

Source temperature is, of course, an important parameter in the mechanism of Direct-EI interface and its effect must be evaluated carefully. The heat distribution throughout the solute must be quick enough to avoid thermal decomposition of the most delicate analytes. A deeper discussion on this topic is reported elsewhere [21]. The right combination of solute mass and temperature constitute the guidelines for a proper analysis and has to be evaluated case by case. For our compounds selection we found that $250^{\circ} \mathrm{C}$ was the best temperature for obtaining the highest signal and the best quality spectrum.

\section{Liquid Chromatography}

Liquid chromatography was carried out with a Kontron Instrument 420 dual-pump, binary-gradient, conventional HPLC system (Kontron Instrument, Milano, Italy). Microliter and nanoliter flow rates were obtained with a laboratory made splitter that was placed between the pumping system and the injector [22]. This device allows the conversion of near conventional flow rates $(200 \mu \mathrm{L} / \mathrm{min})$, generated by a conventional HPLC 
binary system, into capillary-scale flow rates. A two step splitting of the main stream of solvents generates, for instance, a $2 \mu \mathrm{L} / \mathrm{min}$ mobile phase flow rate with a splitting ratio of 100:1. The splitting device accurately reproduces at lower scale any solvent concentration gradient generated at a higher flow rate in the pumping system. For large volume sample injection, relative to the extremely low mobile phase flow rates used in this work, a Valco injector, equipped with a $60 \mathrm{~nL}$ or $100 \mathrm{~nL}$ internal loop, was employed (Valco, Houston, TX). For flow injection analysis (FIA) experiments two different injection valves were used: An Upchurch Scientific M-435 micro-injection valve (Upchurch Scientific, Oak Harbor, WA) and a Rheodyne 7520 (Rheodyne L. P., Rohnert Park, CA). This injector can deliver as low as 2 $\mathrm{nL}$ of sample into the system. A Spectra $100 \mathrm{UV} / \mathrm{Vis}$ detector (Spectra-Physics, San Jose, CA) was placed on-line between the chromatographic column and the HPLC/MS interface for additional peak detection. The sample cell was obtained from the same fused silica capillary tubing used as a connector between the column and the in-source micro-nebulizer eliminating a little segment of the opaque polymide protection. A LC Packings $75 \mu \mathrm{m}$ i.d. column (LC Packings, Amsterdam, The Netherlands) packed with C18, $3 \mu \mathrm{m}$ particles, was used for the chromatographic separations. Acetonitrile was used as organic solvent in the mobile phase. Acetonitrile was preferred to methanol because of its lower viscosity, a parameter that is crucial in microHPLC. In order to achieve a correct solute focusing at the head of the column in large volume injection conditions, every sample solution was prepared with a high percentage of water.

\section{Results and Discussion}

In the Direct-EI, each instrument becomes the natural completion of the other without limitation of the specific potential of each. In addition, liquid chromatography can take advantage of the added specificity offered by the electron ionization detection and mass spectrometry can benefit from a simple and reliable inlet system for liquid samples.

For the evaluation of the performance of the $\mathrm{Di}$ rect-EI interface we selected a few parameters that, in our opinion, are crucial for a successful integration of HPLC and MS. They are: Limit of detection, linearity of response, reproducibility, and separation compatibility. Several compounds, with diverse chemical properties and belonging to different fields of application, were selected for this evaluation. All of them have a limited molecular weight to suit electron ionization conditions and are perfectly amenable to HPLC. The entire set of experiments for the evaluation of the performance of the Direct-EI interface were carried out at different flow rates ranging between 0.3 and $1.5 \mu \mathrm{L} / \mathrm{min}$. Modern micro-HPLC allows efficient separation at less than 100 $\mathrm{nL} / \mathrm{min}$. Several LC companies now offer specific products for gradient micro-HPLC and a complete line of
Table 1. Limits of detection (LODs) in nanograms

\begin{tabular}{lcc}
\hline & SIM LODs & Scan LODs \\
\hline \hline Caffeine & 0.1 & 0.8 \\
Propanil & 0.05 & 1.0 \\
Vitamin K & 0.1 & 1.5 \\
\hline
\end{tabular}

capillary columns. In our work, for generation of gradients at micro-scale flow rate, we opted for a lab-made splitting device that provides good performance at a lower cost.

\section{Limit of Detection}

The limit of detection (LOD), defined as the minimum amount of material detectable by the instrumentation, is one of the most valued features of any analytical instrumentation. It depends mostly on a good signalto-noise ratio rather than solely on the intensity of the signal. In a liquid chromatography-mass spectrometry system the limit of detection depends on the combination of two different factors. The first one is related to the efficiency of the HPLC separation: A narrower peak improves signal-to-noise ratio reducing the effect of the chemical and electronic background. The second factor is related to the mass spectrometer response, and due to its intrinsic complexity, may vary even further. The electron ionization typical efficiency (approximately $0.1 \%$ ) is lower than API or other soft ionization techniques, but conversely, it offers structural information from fragmentation, a feature that surely is worth a little trade-off in sensitivity. It is noteworthy to point out that the interface itself cannot improve LOD but it can offer the ideal basis for maximizing the signal generation. As a matter of fact, Direct-EI improves sample transport and offers picogram level detection similar to a conventional gas chromatography-mass spectrometry (GC/MS) apparatus.

Electron ionization presents two independent levels of detection limits: One is obtained when operating in the selected ion monitoring (SIM) mode and, since the lowest LOD is obtained with this mode, it is considered mostly for quantification purposes. Alternatively, the LOD calculated in full scan acquisition is evaluated mostly for qualitative investigations. It is the latter that is our principal focus. Any little gain in the full spectrum signal obtained from the minimum amount of analyte is particularly welcome in this context. In fact, the strength of this interface is the ability to offer a characteristic mass spectrum to a variety of LC applications. We use different criteria for the calculation of these limits: In SIM the limit of detection is calculated as the signal generated by the mass spectrometer that exceeds the mean value of the background noise by $6 \sigma$, where $\sigma$ is the standard deviation of the baseline; in full scan mode the limit is represented by the least amount of material capable of generating an interpretable mass spectrum. Table 1 provides results of the performance 

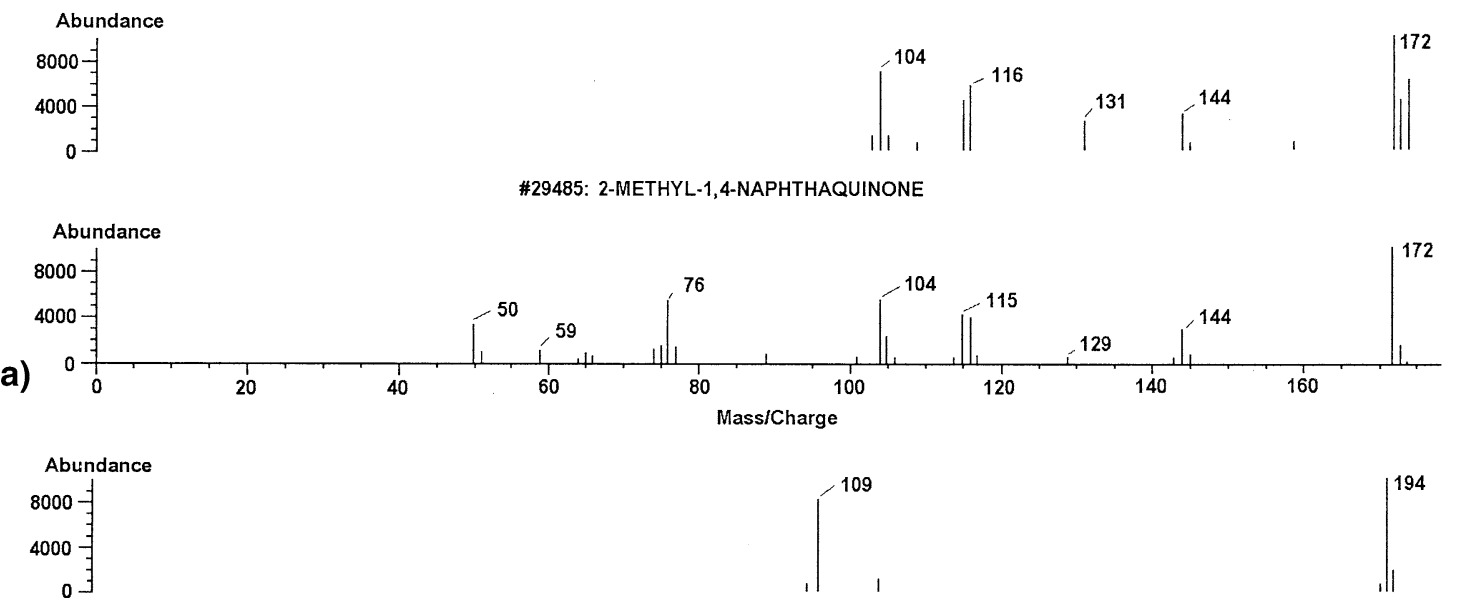

$\# 123308$ : Caffeine

(b)
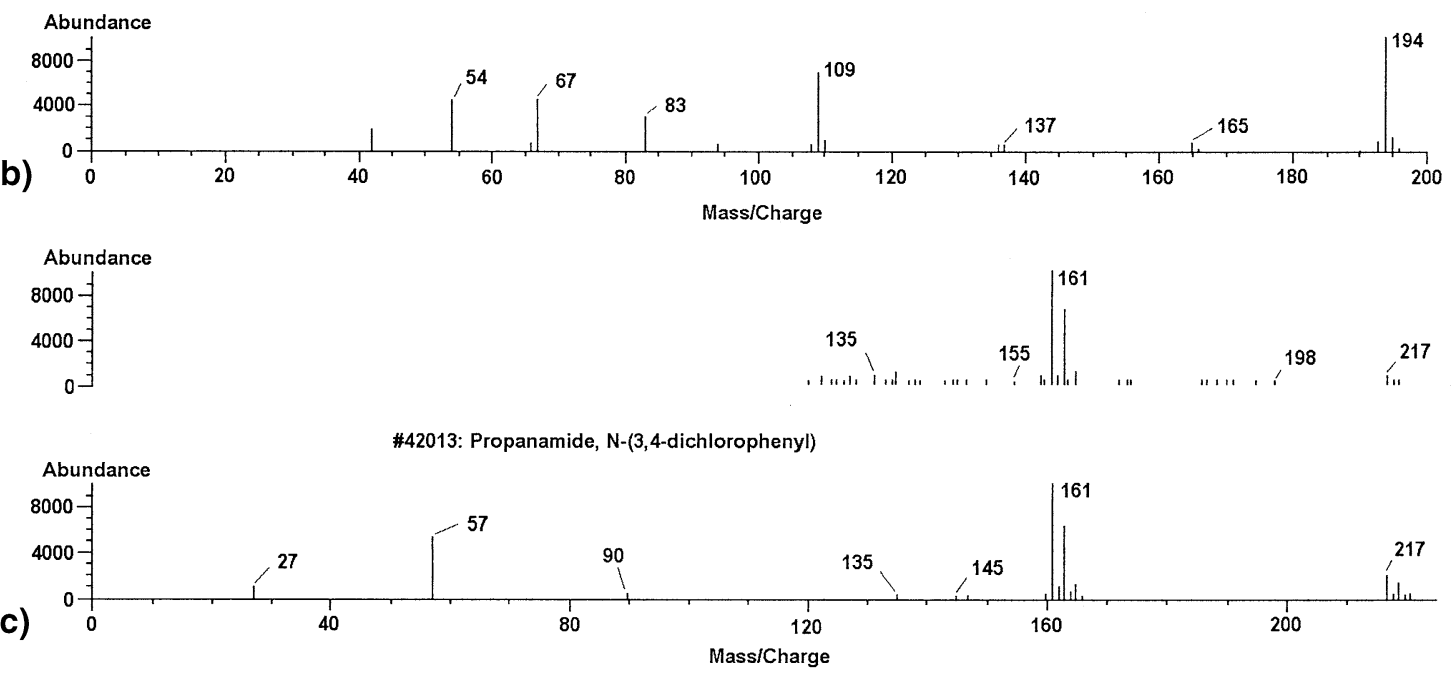

Figure 5. Comparison between recorded (top) and Wiley library (bottom) mass spectra (5 ng injected). (a) vitamin $\mathrm{K}$, (b) caffeine, (c) propanil.

of the Direct-EI interface in terms of LOD. In this case, we used three test compounds, caffeine, propanil, and 2-methyl-1,4-naphthaquinone (vitamin K). Propanil is a propionalide pesticide. All the compounds were eluted through the column under gradient conditions (from 5 to $90 \%$ acetonitrile in $20 \mathrm{~min}$ at a flow rate of 300 $\mathrm{nL} / \mathrm{min}$ ) to better simulate a real world application. These limits of detection represent the best result obtained in electron ionization from a liquid matrix to date. The values reported are appropriate even for ultra-trace level applications.

One of the most important features of this interface is the quality of the electron ionization mass spectra that can be generated. This capability was evaluated by comparing the mass spectra of the three test compounds with those reported in the Wiley electronic library as results after a searching procedure. In Figure 5 we show the average mass spectrum acquired for each test compound close to its limit of detection (5 $\mathrm{ng}$ injected). As clearly seen in the figure, all the spectra are very similar to those of the library. The mass range was intentionally limited to above $\mathrm{m} / \mathrm{z} 100$ to reject most of the chemical noise and enhance signal response. In spite of this, the limited range did not affect the electronic library comparison, giving the following matching quality results: Caffeine $98 \%$, propanil $97 \%$, vitamin K 95\%. Mass-to-charge ratios and relative abundances reproduce very well those reported in literature proving the effectiveness of the direct interface in providing qualitative information.

\section{Reproducibility and Linearity}

The range of the linearity response is a drawback of several HPLC/MS interfacing devices. The problem usually arises from an unreliable solute transport process, which in most cases cannot be corrected. As already mentioned, the Direct-EI interface is physically linked to the HPLC column and every aliquot of the sample is introduced into the ion source. Therefore, a linear response over a wide range of concentrations was expected. Calibration experiments were performed over a wide range of concentrations for the same three test compounds, starting near the detection limit and ex- 
tending up two orders of magnitude (2-200 ng). Five different concentrations were analyzed, with five replicates for each one. The experiment was carried out by FIA using a mobile phase of water and acetonitrile in equal proportions. The flow rate was set at $500 \mathrm{~nL} / \mathrm{min}$ and the mass spectral acquisition was carried out in SIM mode with single ion detection. The standard solutions were prepared in methanol. Linear regression equations and mean standard deviation data were calculated. The linear regression equations and correlation coefficients obtained are:

- Caffeine: $y=-5.01+1.77 x$

$$
\begin{aligned}
& r=0.9996 \\
& r=0.9995 \\
& r=0.9982
\end{aligned}
$$

The correlation coefficients are all close to unity indicating good linearity over the measured range. Sensitivity, expressed as the angular coefficient of the linear regression equation, was highest for Vitamin $\mathrm{K}$ with a value of 4.30 area units/ng and lowest for caffeine where a value of 1.77 area units/ng was obtained.

Reproducibility was evaluated throughout the day from a series of consecutive injections. One ng of each compound was injected into the system and detected in SIM mode. Response variations were very limited as indicated by the good relative standard deviation values (RSD) calculated for the selected compounds:

- Caffeine $\pm 8 \%$

- Propanil $\pm 3.8 \%$

- Vitamin $\mathrm{K} \pm 7.4 \%$

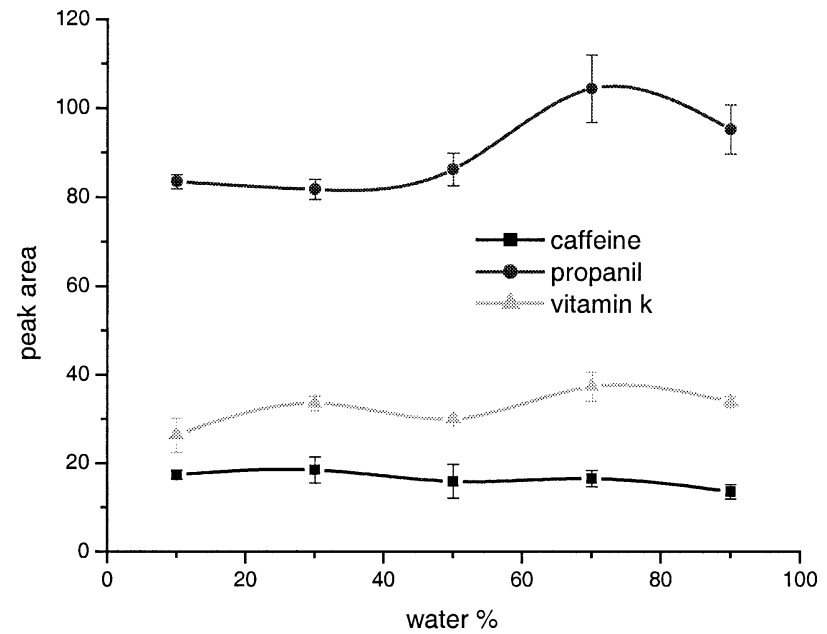

Figure 6. Signal response versus mobile composition was evaluated for the three test compounds. The experiment was carried out in SIM mode injecting in FIA $10 \mathrm{ng}$ of each analyte using different mobile phase compositions.

\section{Separation Compatibility}

This section is dedicated to the evaluation of the interface potential in different chromatographic conditions and for different analytes. The investigation cannot be exhaustive in this context, but may help to underline the positive behavior of this interface in some practical applications. In HPLC/MS, the most important variable is represented by the mobile phase composition. In addition, mobile phase polarity, viscosity, volatility, and other chemical or physical properties may change drastically during solvent gradient analysis and affect

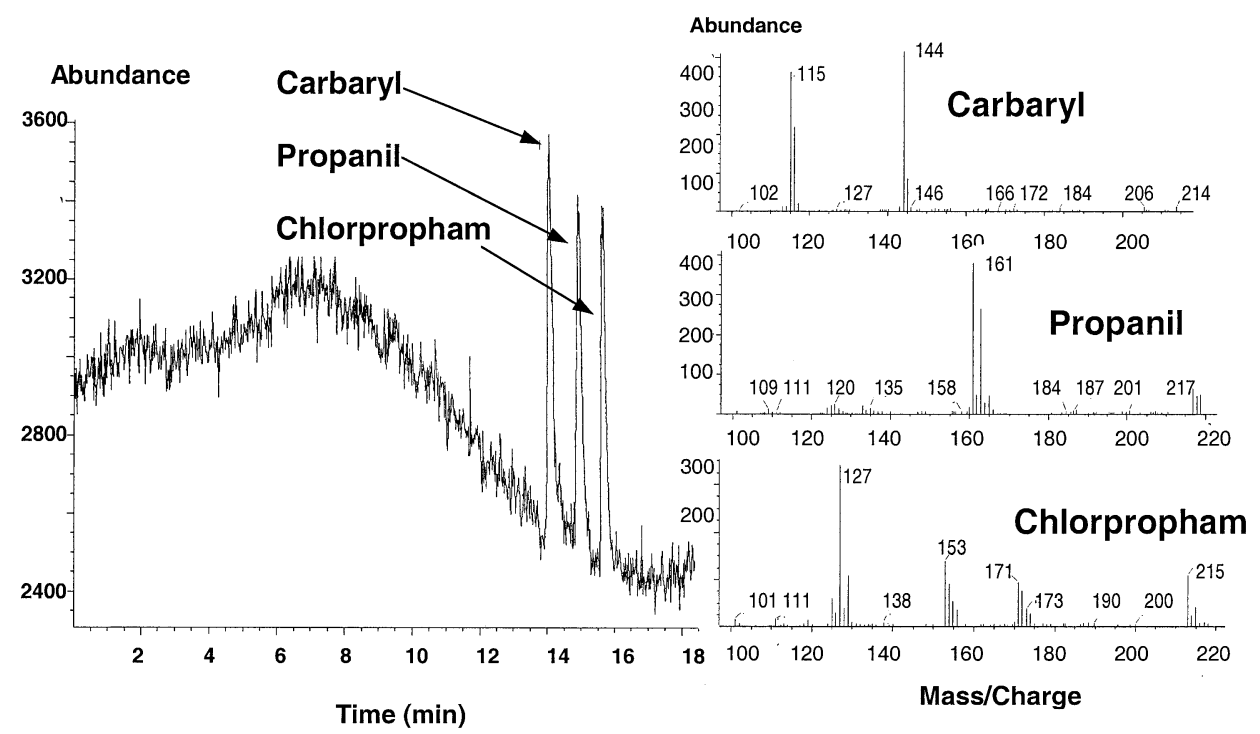

Figure 7. Full spectrum profile and mass spectra relative to the separation of three pesticides (5 ng injected): Carbaryl, propanil, and chlorpropham. The separation was obtained in gradient condition using a reversed phase, C18 nano-column. The flow rate was $300 \mathrm{~nL} / \mathrm{min}$ and mobile phase composition was changed from 10 to $80 \%$ acetonitrile in water in $20 \mathrm{~min}$. The mass spectrometer was operating in scan mode from 100 to $220 \mathrm{u} 5 \mathrm{ng}$ of each compound were injected into the system using a $50 \mathrm{ng} / \mu \mathrm{L}$ solution and injecting $100 \mathrm{~nL}$. 

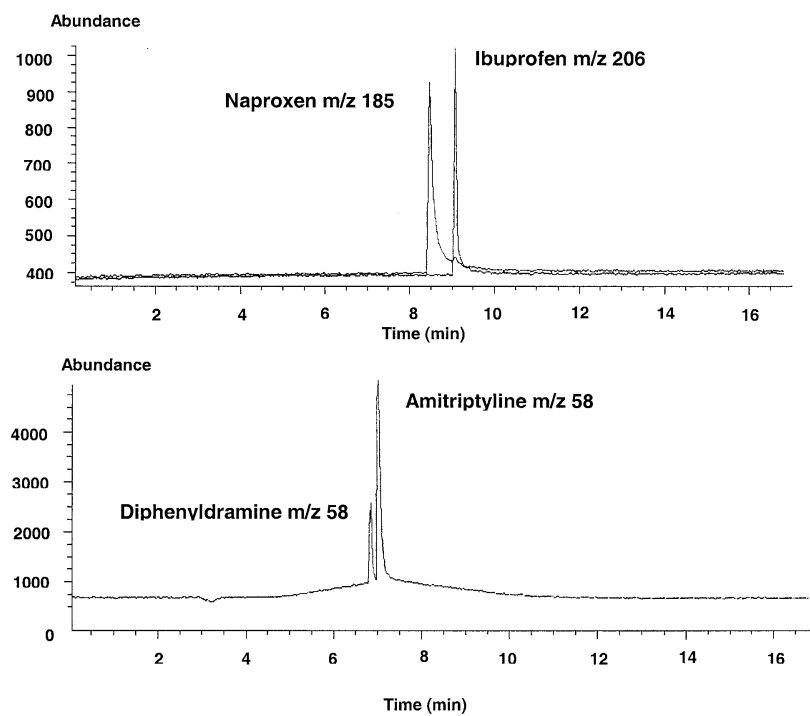

Figure 8. Ion profiles relative to the separation of four antiinflammatory drugs: Diphenhyldramyne, amitriptyline, naproxen, and ibuprofen. The mobile phase was modified with $0.1 \%$ formic acid and its composition was changed from 10 to $80 \%$ acetonitrile in $20 \mathrm{~min}$. A reversed phase C18 nano-column and a flow rate of $300 \mathrm{~nL} / \mathrm{min}$ were used. The mass spectrometer was operating in SIM mode monitoring $m / z 58,185$, and 206 . The ion at $\mathrm{m} / \mathrm{z} 58$ was used for the detection diphenhyldramyne and amitriptyline, $\mathrm{m} / \mathrm{z} 185$ for naproxen, and $\mathrm{m} / \mathrm{z} 206$ for ibuprofen. $750 \mathrm{pg}$ of each analyte were injected using a $7.5 \mathrm{ng} / \mu \mathrm{L}$ solution and a $100 \mathrm{~nL}$ internal loop.

the overall instrument performance. Various mobile phase modifiers, often necessary for solving complex separations, may further worsen the situation. To test the interface behavior in different chromatographic conditions, we recorded the signal response of our selected compounds for various ratios of water and acetonitrile between 10 and 90\%. The experiment simulates a gradient analysis in reversed phase conditions, by far the most popular HPLC technique. The amount injected, $10 \mathrm{ng}$, was identical for the three test compounds and the acquisition was in the SIM mode. The plots are shown in Figure 6. The purpose of the experiment was to simulate a reversed phase solvent gradient analysis and to monitor the behavior of the interface in controlled conditions. Data was obtained over the range of $10 \% / 90 \%$ to $90 \% / 10 \%$ water/acetonitrile, in $20 \%$ steps. Two compounds, propanil (filled circle) and vitamin K (filled triangle), performed slightly better at a higher concentration of water while caffeine (filled square) gave a flatter response. As clearly indicated in the figure, the Direct-EI interface is not influenced by any variation in the mobile phase composition and its response remains flat for all compounds. This may be explained by the marginal role played by the solvent in this specific interfacing process. The most volatile fraction of the liquid mixture, represented by the solvents, is quickly removed leaving the solute behind in the ion volume for ionization.

Two real world applications are reported in Figures 7 and 8 . The first one, of environmental interest, shows the separation of three pesticides, carbaryl, propanil, and chlorpropham. The separation was obtained in gradient condition using a reversed phase, C18 nanocolumn. The flow rate was $300 \mathrm{~nL} / \mathrm{min}$ and mobile phase composition was changed from 10 to $80 \%$ acetonitrile in water in $20 \mathrm{~min}$. The mass spectrometer was operating in scan mode from 100 to $220 \mathrm{u}$. Five ng of each compound were injected into the system using a 50 $\mathrm{ng} / \mu \mathrm{L}$ solution and injecting $100 \mathrm{~nL}$. The standard mixture was prepared using the starting mobile phase composition. The second example, reported in Figure 8, of pharmaceutical interest, shows the ion profile relative to the separation of four anti-inflammatory drugs, diphenhyldramyne, amitriptyline, naproxen, and ibuprofen. The mobile phase was modified with $0.1 \%$ formic acid and its composition was changed from 10 to $80 \%$ acetonitrile in $20 \mathrm{~min}$. Also in this case we used a reversed phase $\mathrm{C} 18$ nano-column and a flow rate of 300 $\mathrm{nL} / \mathrm{min}$. The mass spectrometer was operating in SIM mode monitoring $\mathrm{m} / \mathrm{z} 58,185$, and 206. The ion at $\mathrm{m} / \mathrm{z} 58$ was used for the detection diphenhyldramyne and amitriptyline, $\mathrm{m} / \mathrm{z} 185$ for naproxen, and $\mathrm{m} / \mathrm{z} 206$ for ibuprofen. $750 \mathrm{pg}$ of each analyte were injected using a $7.5 \mathrm{ng} / \mu \mathrm{L}$ solution and a $100 \mathrm{~nL}$ internal loop. As in the previous example, the standard solution was prepared using the same initial mobile phase mixture.

This work demonstrates that it is possible to use a simple and effective HPLC/MS interface for a multitude of applications. Direct-EI offers to the analyst a powerful tool for the identification of several compounds in a liquid phase. Being based on the electron ionization technique, the new interface allows a direct comparison of any recorded spectrum with tens of thousands electronically archived spectra for an easier identification of small to medium sized molecules at a level of sensitivity not far from that offered by an API interface.

\section{Acknowledgments}

The authors would like to thank the following companies: Rheodyne L.P., Rohnert Park, CA for providing the 7520 micro-injector and Upchurch Scientific, Oak Harbor, WA for providing the M-435 micro injection valve.

\section{References}

1. Dole, M.; Mack, L. L.; Hines, R. I.; Mobley, R. C.; Ferguson, L. D. M.; Alice, B. J. Chem. Phys. 1968, 49, 2240.

2. Yamashita, M.; Fenn, J. B. J. Phys. Chem. 1984, 88, 4451.

3. Horning, E. C.; Carroll, D. I.; Dzidic, I.; Haegele, K. D.; Horning, M. G.; Stillwell, R. N. J. Chromatogr. Sci. 1974, 12, 725.

4. Fenn, J. B. J. Am. Soc. Mass Spectrom. 1993, 4, 524.

5. Cappiello, A. Mass Spectrometry Reviews 1996, 15, 283.

6. Cappiello, A.; Famiglini, G. J. Am. Soc. Mass Spectrom. 1998, 9, 993.

7. Willoughby, R. C.; Browner, R. F. Anal. Chem. 1984, 56, 2626.

8. Cappiello, A.; Bruner, F. Anal. Chem. 1993, 65, 1281.

9. Cappiello, A.; Balogh, M.; Famiglini, G.; Mangani, F.; Palma, P. Anal. Chem. 2000, 72, 3841. 
10. Kientz, C. E.; Huist, A. G.; De Jong, A. L.; Wils, E. R. J. Anal. Chem. 1996, 68, 675.

11. Dijkstra, R.; Van Baar, B. L. M.; Kientz, C. E.; Niessen, W. M. A.; Brinkman, U. A. Rapid Commun. Mass Spectrom. 1998, 12,5 .

12. Cappiello, A.; Famiglini, G.; Palma, P.; Mangani F. Proceedings of the 15th International Mass Spectrometry Conference; Barcelona, Spain, August, 2000 p 389.

13. Vesta, M.; Blakle, C.; Ryan, P.; Futrell, J. H. Adv. Mass Spectrom. 1974, 6, 781.

14. Arpino, P. J.; Krien, P.; Vajta, S.; Devant, G. J. Chromatogr. 1981, 203, 117.
15. Tijssen, R.; Bleumer, J. P. A.; Smit, A. L. C.; Van Kreveld, M. E. J. Chromatogr. 1981, 218, 137.

16. Shäfer, K. H.; Levsen, K. J. Chromatogr. 1981, 206, 245.

17. Mauchamp, B. J. Chromatogr. 1982, 236, 17.

18. Arpino, P. J.; Beaugrand, D. Int. J. Mass Spectrom. Ion Proc. 1985, 64, 275.

19. De Wit, J. S. M.; Tomer, K. B.; Jorgenson, J. W. J. Chromatogr. 1989, 462, 365.

20. Bruins, A. P.; Drenth, B. F. H. J. Chromatogr. 1983, 271, 71.

21. Cappiello, A.; Famiglini, G. Anal. Chem. 1995, 67, 412.

22. Berloni, A.; Cappiello, A.; Famiglini, G.; Palma, P. Chromatographia 1994, 39, 279. 